\title{
COVID-19 e suas manifestações no sistema nervoso
}

\author{
COVID-19 and its nervous system manifestations \\ COVID-19 y sus manifestaciones en el sistema nervioso
}

Guilherme Ferreira Santos Silva1*, Samuel Ramos Rabelo ${ }^{1}$, Taline Cristine de Sena Cardoso1, Esley Ruas Alkimim ${ }^{1}$, Claudiney Cordeiro Arruda ${ }^{1}$, Rafael Bessa Fleming ${ }^{2}$, Maria Tereza Castro de Figueiredo ${ }^{1}$, Lucia Dannielly Antunes Faria ${ }^{1}$, Odilon José Monteiro Neto ${ }^{1}$, Marcelo José da Silva de Magalhães ${ }^{1,3,4}$.

\section{RESUMO}

Objetivo: Delimitar as manifestações de aspecto neurológicos provocadas pela infecção pelo Sars-Cov-,2 vírus causador da COVID-19. Revisão Bibliográfica: O SARS-CoV-2 é um vírus predominantemente relacionado a manifestações clínicas no sistema respiratório, mas atualmente exibe evidências consistentes que apontam para o acometimento do sistema nervoso, evidenciando um neurotropismo. Os sintomas neurológicos mais frequentemente relatados são: cefaleia, anosmia, ageusia e ocorrência de eventos cerebrovasculares (AVE). Ademais, a infecção por SARS-CoV-2 também tem sido relacionada como fator de risco para a ocorrência da doença de Alzheimer, Síndrome de Guillain Barré, cefaleias, encefalites, alterações nos níveis de consciência e também à presença de paralisia facial periférica. Acredita-se que pacientes com acometimento neurológico pré-existentes sejam mais propensos a desenvolver complicações nervosas como consequência da infecção por SARS-Cov-2. Considerações finais: O COVID-19 pode causar uma gama de doenças neurológicas. Faz-se necessário a realização de estudos acerca do tema a fim de esclarecer melhor os achados e conduta clínica a ser adotada.

Palavras-chave: Perturbações neurológicas, COVID-19, Manifestações neurológicas.

\begin{abstract}
Objective: To delimit the neurological manifestations caused by infection by Sars-Cov-, 2 virus that causes COVID-19. Bibliographic review: SARS-CoV-2 is a virus predominantly related to clinical manifestations in the respiratory system, but currently displays consistent evidence that points to the involvement of the nervous system, showing a neurotropism. The most frequently reported neurological symptoms are: headache, anosmia, ageusia and occurrence of cerebrovascular events (CVA). In addition, SARS-CoV-2 infection has also been linked as a risk factor for the occurrence of Alzheimer's disease, Guillain Barré syndrome, headache, encephalitis, changes in levels of consciousness and also the presence of peripheral facial paralysis. It is believed that patients with pre-existing neurological impairment are more likely to develop nerve complications as a result of SARS-Cov-2 infection. Final considerations: COVID-19 can cause a range of neurological diseases. It is necessary to carry out studies on the topic in order to better clarify the findings and clinical conduct to be adopted.
\end{abstract}

Keywords: Nervous system diseases, Coronavirus infections, Neurologic manifestations.

\section{RESUMEN}

Objetivo: Delimitar las manifestaciones neurológicas provocadas por la infección por el virus Sars-Cov-,2 causante del COVID-19. Revisión bibliográfica: EI SARS-CoV-2 es un virus predominantemente relacionado con manifestaciones clínicas en el sistema respiratorio, pero actualmente muestra evidencia consistente que

${ }^{1}$ Centro Universitário FUNORTE (UNIFUNORTE), Montes Claros - MG. *E-mail: guilferr94@gmail.com

2 Faculdade de Minas (FAMINAS), Belo Horizonte - MG.

${ }^{3}$ Hospital Vila da Serra-Nova Lima, Nova Lima - MG.

${ }^{4}$ Hospital Aroldo Tourinho, Montes Claros - MG. 
apunta a la afectación del sistema nervioso, mostrando un neurotropismo. Los síntomas neurológicos notificados con mayor frecuencia son: cefalea, anosmia, ageusia y aparición de eventos cerebrovasculares (ACV). Además, la infección por SARS-CoV-2 también se ha relacionado como factor de riesgo para la aparición de la enfermedad de Alzheimer, síndrome de Guillain Barré, dolor de cabeza, encefalitis, cambios en los niveles de conciencia y también la presencia de parálisis facial periférica. Se cree que los pacientes con deterioro neurológico preexistente tienen más probabilidades de desarrollar complicaciones nerviosas como resultado de la infección por SARS-Cov-2. Consideraciones finales: COVID-19 puede causar una variedad de enfermedades neurológicas. Es necesario realizar estudios sobre el tema para aclarar mejor los hallazgos y la conducta clínica a adoptar.

Palabras Clave: Enfermedades del sistema nervioso, Infecciones por coronavirus, Manifestaciones neurológicas.

\section{INTRODUÇÃO}

Descrita inicialmente em dezembro de 2019 em Wuhan, na China, a COVID-19 é uma doença complexa, multissistêmica, que ocorre em função da infecção pelo vírus denominado severe acute respiratory syndrome - coronavirus 2 (SARS-CoV-2), declarado pandêmico em 11 de março de 2020 pela Organização Mundial da Saúde (OMS). A maioria dos pacientes são assintomáticos, e as manifestações mais comuns são febre, tosse, odinofagia, cefaleia, fadiga e mialgia; podem ocorrer também desconfortos gastrointestinais, como diarreia e vômitos (GUAN WJ, et al., 2020; CHU H, et al., 2020; DE FELICE FG, et al., 2020; WU Y, et al., 2020).

Ainda que a doença do coronavírus COVID-19, síndrome desencadeada a partir da infecção pelo SARSCoV- 2, seja caracterizada principalmente por comprometer o trato respiratório, atualmente existem evidências que apontam também para o acometimento dos sistemas cardiovascular, digestório e renal e, mais recentemente, para o comprometimento do sistema nervoso (KOCHI AN, et al., 2020; LING L, et al.,2020).

Embora sejam dados preliminares, Mao L, et al. (2020); verificaram que $36,4 \%$ dos pacientes hospitalizados apresentaram manifestações neurológicas, a maioria delas de caráter agudo e início precoce, em função da infecção por SARS-CoV-2, sugerindo envolvimento direto do sistema nervoso pela infecção viral; esses pacientes, em geral, eram mais idosos e portadores de comorbidades como hipertensão, diabetes mellitus e dislipidemia (BEYROUTI R, et al., 2020; JIN H, et al., 2020; PAPA SM, et al., 2020; PLEASURE SJ, et al., 2020).

Acredita-se que a infecção viral, associada à excessiva ativação do sistema imunológico do hospedeiro e o dano hipóxico em função do comprometimento pulmonar, possa contribuir para a instalação e persistência de déficits neurológicos. Portanto, faz-se necessária uma análise minuciosa de pacientes que cursam com o quadro de COVID-19, atentando-se para cefaleias, distúrbios de consciência e outros sinais patológicos que, porventura, possam vir a se apresentar (WU Y, et al.,2020).

Sendo assim, é objetivo deste trabalho abordar as principais manifestações neurológicas da COVID-19, para melhor compreensão da doença por profissionais e por acadêmicos da área da saúde, expondo-se a epidemiologia, a fisiopatologia e os mecanismos pelos quais ocorrem essas manifestações. Esta revisão narrativa da literatura pretende ainda, elucidar para o leitor, de forma clara e objetiva, as diversas formas de acometimento do sistema nervoso pelo SARS-CoV-2 e as complicações mais frequentemente relatadas, de modo a identificar lacunas de conhecimento importantes que possam subsidiar novas pesquisas.

\section{REVISÃO BIBLIOGRÁFICA}

Após analisar a literatura atual; um artigo com ênfase em AVEs (uma série de casos envolvendo seis pacientes no National Hospital for Neurology and Neurosurgery em Queen Square, na cidade de Londres); dois artigos com ênfase em encefalite (uma série de casos envolvendo oito pacientes, e um relato de caso); dois artigos com ênfase em disfunções olfativas e gustativas (dois relatos de caso); dois artigos com ênfase 
em manifestações neurológicas gerais, como cefaleia, Alzheimer, etc. (um estudo retrospectivo e observacional envolvendo 144 pacientes internados na cidade de Wuhan, e uma revisão comparativa); um artigo com ênfase em paralisia facial periférica (relato de caso); e dois artigos a respeito da síndrome de Guillain-Barré (ambos os relatos de caso).

Com base na prática clínica e relatos de estudos, acredita-se que pacientes com disfunções neurológicas pré-existentes sejam mais propensos a desenvolver complicações a nível do sistema nervoso como consequência da infecção por Sars-Cov-2 (LOPES J, 2020). A maioria dos indivíduos que cursam com manifestações neurológicas como consequência da infecção pelo Sars-Cov-2 tem acometimento neurológico leve marcadamente pela presença de hipogeusia, hiposmia e cefaleia, com grande maioria evoluindo para a recuperação completa. No entanto, uma pequena parcela, com especial atenção aos idosos com múltiplos fatores de risco (hipertensão, diabetes mellitus, dislipidemia, obesidade, etc.), podem vir a desenvolver sintomas mais graves (DE SOUZA GOMES A, et al., 2020).

Sabe-se que há um envolvimento direto do encéfalo pelo SARS-CoV-2, fatores auto-imunes, inflamação ("Tempestade de citocinas"), efeitos colaterais dos medicamentos e distúrbios metabólicos também estão correlacionados. Na fisiopatologia, mecanismos diretos e indiretos são atribuídos (PAPA SM, et al., 2020; PLEASURE SJ, et al., 2020). Ainda não há muitos estudos experimentais que possam esclarecer melhor o comportamento de disseminação do COVID-19 no sistema nervoso, mas têm sido propostos dois prováveis mecanismos lesivos em função dos quais a COVID-19 pode resultar em acometimento neurológico, sendo eles: lesão por hipóxia ou lesão por mecanismos imunológicos (LOPES, J. 2020)

Pacientes acometidos pela COVID-19 podem cursar com hipóxia grave (GUO YR, et al., 2020). Como discutido por Abdennour L, et al. (2012) no artigo "Interaction brain-lungs", a infecção viral no parênquima pulmonar pode levar a uma inflamação alveolar, comprometendo substancialmente o processo de hematose. Tal distúrbio pode colaborar para hipóxia do sistema nervoso, instalando-se então um processo isquêmico que resulta, principalmente, em distúrbios da circulação cerebral e em edema intersticial, que podem acentuar-se em casos de hipóxia prolongada, evoluindo para quadros de hipertensão intracraniana, de doença cerebrovascular aguda e de deterioração gradual da função cerebral. Nesse sentido, o metabolismo anaeróbico, portanto, promove a formação de metabólitos tóxicos causando mau funcionamento e edema cerebral (LOPES J, 2020).

A infecção por SARS-CoV-2 também é capaz de desencadear uma reação imune excessiva, denominada tempestade de citocinas. Essa tempestade induz o aumento de interleucinas (IL), e estas estariam diretamente relacionadas com a sintomatologia apresentada em todo o organismo, inclusive com os acometimentos do sistema nervoso, por exemplo, aqueles decorrentes da lesão por mecanismo imunológico (MCGONAGLE D, et al.,2020).

Como discutido por Li Y, et al. (2004) e por Bohmwald K, et al. (2018) a presença do vírus no sistema nervoso ativa as células gliais, que passam a secretar IL-6, além de IL-12, IL-15 e TNF-a, resultando em uma hiperativação dos fatores pró-inflamatórios e colaborando para a instalação de lesão e de déficits no sistema neurológico.

Por todos os achados acima mencionados, considera-se a possibilidade do desenvolvimento de processos reativos imunomediados no sistema nervoso central promovendo encefalites, mielites, radiculites, polirradiculoneurites, até mesmo desmielinizantes. O sistema nervoso periférico também não está ileso nesse processo pois muitos pacientes têm apresentado neurites e neurites múltiplas associadas a infecção pelo SARS-CoV-2 (LOPES J, 2020).

\section{Doenças cerebrovasculares}

A ocorrência de doenças cerebrovasculares em pacientes infectados pelo vírus SARS-CoV-2 está relacionada à hiperativação dos fatores pró-inflamatórios, que ocorre concomitantemente com a disfunção dos fatores de coagulação, especialmente o dímero-D, e com as anormalidades plaquetárias. Na infecção por SARS-CoV-2, foi amplamente relatada a ocorrência de tempestades de citocina, marcada principalmente pela 
presença da interleucina 6 (IL-6) favorecendo a ocorrência do extravasamento vascular, ativação do complemento e disseminação da coagulação intravascular o que, por sua vez, colabora para o dano orgânico. (LOPES J, 2020; MUNHOZ RP, et al.,2020; MEHTA P, et al.,2020; JIN H, et al., 2020).

Associadamente a tempestade de citocinas, foram verificados níveis elevados de dímero-D (comum na coagulação vascular disseminada e em casos de trombose) e grave plaquetopenia. Frequentemente se verificam alterações na pressão arterial de pacientes acometidos por SARS-CoV-2, sendo esse processo provavelmente provocado pela capacidade viral de interagir com os receptores de angiotensina 2 expressos no endotélio capilar da rede vascular que drena e irriga o sistema nervoso, colaborando para o aumento da probabilidade de ocorrência de hemorragia cerebral (LOPES J, 2020; MUNHOZ RP, et al.,2020; MEHTA P, et al.,2020; JIN H, et al., 2020).

Os acidentes vasculares encefálicos (AVEs) decorrentes da infecção pelo SARS-CoV-2 geralmente ocorrem alguns dias após o contágio e são mais comuns em pacientes com a forma grave da COVID-19 (WANG Z, et al., 2020). O mais comum é que esses acidentes vasculares ocorram em grandes vasos, podendo levar a uma grande variedade de sintomas. Em um estudo que analisou a ocorrência de AVEs isquêmicos decorrentes da COVID-19 em seis pacientes, as artérias mais afetadas foram: artéria vertebral esquerda, artéria cerebelar inferior posterior esquerda, artéria cerebral posterior esquerda, artéria cerebral média direita e artéria basilar, o que evidencia a grande variedade de artérias acometidas (WANG Z, et al, 2020; BEYROUTI R, et al.,2020).

Os principais sintomas que esses pacientes apresentaram foram: dificuldade na coordenação de palavras, incoordenação motora bilateral, hemianopsia homônima, confusão mental, rebaixamento do nível de consciência, disartria, hemiparesias, paralisia facial e afasia. $O$ tratamento desses pacientes variou entre antibioticoterapia, suplementação de oxigênio, tratamento de suporte e medicamentos anticoagulantes (BEYROUTI R, et al., 2020).

Ainda não se sabe, com certeza, se a ocorrência de AVEs hemorrágicos está diretamente ligada à infecção pelo SARS-CoV-2, visto que, até o presente momento, não foram relatados muitos casos em que as duas doenças ocorreram simultaneamente. Entretanto, é possível observar que, na maioria dos casos em que o paciente estava infectado e sofreu um AVE hemorrágico, o indivíduo possuía comorbidades prévias, como hipertensão arterial sistêmica. Essa predisposição possivelmente está relacionada com a regulação para a redução da enzima ECA-2 pelo SARS-CoV-2, aumentando-se os níveis de angiotensina 2, os quais normalmente já estão mais elevados em pessoas hipertensas. Essa elevação predispõe sangramentos e, evidentemente, AVEs hemorrágicos. Não foi possível observar, nos artigos analisados, padrão claro de acometimento anatômico dos AVEs hemorrágicos (WANG Z, et al., 2020).

\section{Encefalite}

A encefalite ocorre pela instalação de lesão inflamatória no parênquima cerebral, desencadeada por patógenos, e pode levar à ocorrência de convulsões e de distúrbios de consciência, além de cefaleia. A encefalite por infecção da COVID-19 passou a ter base sólida após verificação de presença do SARS-CoV-2 no liquor (XIANG P, et al., 2020; ELLUL MA e SOLOMON T, 2018; WANG Z, et al., 2020).

Já é de conhecimento geral que determinados vírus podem provocar encefalite aguda, sendo que, em alguns casos de pacientes com COVID-19 e encefalite, foi possível demonstrar a presença do SARS-CoV-2 no liquor (MONTALVAN V, et al., 2020).

Xiang P, et al. (2020) descreveram o caso de um paciente que evoluiu de sintomas inespecíficos flu-like para quadro grave de encefalite, chegando ao ambiente hospitalar com pontuação 6 na Escala de Coma de Glasgow (ECG). Além disso, foi possível observar na ressonância magnética (RM) de crânio, realizada 20 horas após a admissão do paciente, hiperintensidade na parede do corno inferior do ventrículo lateral direito, na sequência DWI. Na sequência FLAIR, puderam ser observadas alterações de sinais hiperintensos no lobo temporal direito e no hipocampo, com leve atrofia hipocampal. Um diagnóstico diferencial importante apontado foi a esclerose hipocampal, que geralmente acompanha a encefalopatia pós- convulsiva. 
Ellul MA, et al. (2020) analisaram oito pacientes que apresentaram encefalite após infecção pelo SARSCoV- 2, sendo que a maioria deles evoluiu com encefalite após cerca de 17 dias do início dos sintomas respiratórios. Os principais sintomas neurológicos apresentados pelos pacientes foram: irritabilidade, rebaixamento do nível de consciência, rigidez de nuca, convulsões, sintomas psicóticos e alterações motoras, como ataxia de marcha. Nenhum tratamento específico foi realizado nos pacientes, feita apenas a administração de anticonvulsivantes, antivirais e antibióticos.

O padrão liquórico dos pacientes infectados pelo SARS-CoV-2 e que desenvolveram a doença foi bastante diversificado, indo desde um padrão de normalidade até um padrão com aumento de celularidade e com presença desse vírus no liquor (MORIGUCHIT T, et al., 2020; VANDERVORST F, et al., 2020).

\section{Cefaleias e alterações do nível de consciência}

Cerca de $40 \%$ dos infectados que apresentaram COVID-19 cursaram com cefaleia, alterações do nível de consciência e sintomas de disfunção cerebral. A ocorrência desses sintomas em função da infecção por SARS-CoV-2 está relacionada à presença de encefalite e de encefalopatias (MAO L, et al., 2020).

$\mathrm{Na}$ encefalopatia, é possível identificar edema cerebral, mas sem a presença de conteúdo inflamatório. Pode ser desencadeada e intensificada pela hipóxia do sistema nervoso e pela toxemia sistêmica. Pacientes que apresentam quadro clínico com melhor prognóstico geralmente cursam com cefaleia, disfonia, transtornos mentais e delírios, enquanto pacientes com pior prognóstico geralmente evoluem com desorientação, paralisias, perda da consciência e coma. Nesse sentido, o mecanismo de apresentação dessa doença em quadros de COVID-19 ocorre possivelmente pela hipoxemia e pela viremia grave (LENNON JC, 2020; MORIGUCHIT T, et al., 2020).

\section{Anosmia e ageusia}

Sharifian-Dorche M, et al. (2020) verificaram que a anosmia e a ageusia foram relatadas em 3.730 pacientes, em 28 estudos publicados, sendo mais frequentes entre mulheres e jovens não hospitalizados. Acredita-se que o mecanismo de acometimento desses dois sintomas esteja relacionado à ação do SARSCoV-2 nos receptores ECA2 expressos nas células que compõem o nervo olfatório e também a via gustativa. A interação desses dois vetores leva à inativação dos canais de membrana, produzindo então alterações na percepção sensorial de odores e sabores (VAIRA LA, et al., 2020)

No mesmo trabalho supracitado, foi observado que a anosmia típica da infecção pelo SARS-CoV-2 geralmente não é acompanhada de coriza ou rinite, sintomas típicos de outras síndromes gripais (SHARIFIAN-DORCHE M, et al., 2020). Além disso, o mesmo artigo afirma que a anosmia é uma manifestação clínica típica do início da manifestação da doença. Por outro lado, Lee JM, Lee CJ (2020) relataram um caso de uma paciente infectada pelo SARS- CoV-2 que apresentou anosmia cerca de 20 dias após o diagnóstico de COVID-19; os autores explicam que essa ocorrência atípica provavelmente se deu em razão de um medicamento inibidor de TNF-alfa que a paciente utiliza para controle da espondilite anquilosante.

Pode-se observar também que a presença de ageusia acompanha a anosmia, haja vista a relação intrínseca entre paladar e olfato; além disso, essas manifestações, ao contrário das trabalhadas anteriormente, pertencem ao sistema nervoso periférico (SHARIFIAN-DORCHE M, et al., 2020).

\section{Demência de Alzheimer (DA)}

Lennon JC (2020) buscou fazer um paralelo entre a infecção por SARS-CoV-2 e o risco de desenvolvimento de Alzheimer. Consoante a publicação, as alterações linfocíticas presentes na COVID-19 podem favorecer o surgimento do quadro de Alzheimer.

Mao L, et al. (2020) verificaram que a contagem de neutrófilos em pacientes que possuíam lesão muscular e COVID-19 positiva internados em Wuhan, China, apresentava-se elevada em comparação com aqueles sem lesão. Embora o neutrófilo seja um mecanismo importante na manutenção da homeostase, ao agir como um mediador da resposta imune, a inflamação pode cursar com danos teciduais, colaborando para o aparecimento de um fenótipo tóxico no sistema nervoso. Esse fenótipo pode vir a favorecer a inserção de 
moléculas que aumentem os riscos de ocorrência da doença de Alzheimer. Esses autores inferem que existe associação entre as complicações neurológicas e imunológicas da COVID-19 e aquelas que afetam a neurodegeneração e a patogênese da DA. Mais estudos são relevantes, com o intuito de estabelecer um melhor entendimento sobre o assunto (LENNON JC, 2020).

\section{Síndrome de Guillain-Barré}

A Síndrome de Guillain Barré (SGB) é uma doença autoimune de caráter agudo que acomete os nervos periféricos e as raízes nervosas, sendo entendida como uma polirradiculoneuropatia. Esta condição está associada a quadros infecciosos, principalmente infecções do trato gastrointestinal e infecções pulmonares. Nesse sentido, estudos apontam para relação da infecção por Sars-Cov-2 com a ocorrência da SGB (LOPES J, 2020).

Zhao H, et al. (2020) relatam o primeiro caso de paciente que apresentou síndrome de Guillain-Barré como manifestação da COVID-19. Essa paciente apresentou paresia aguda de membros inferiores e fadiga extrema como manifestação primária da doença. No momento da admissão hospitalar, ela não apresentava sintomas flu-like. O tratamento foi realizado por meio de antivirais e imunoglobulina, evoluindo a paciente sem sintomas.

Camdessanche JP, et al. (2020) descreveram o caso de um paciente de 64 anos de idade que se apresentou ao hospital após ocorrência de queda e lesão do ombro esquerdo. Febre e tosse surgiram dois dias após a internação, com diagnóstico confirmado de COVID-19 após teste. O paciente evoluiu com agravamento do quadro, necessitando de oxigênio suplementar. Foi tratado com paracetamol, com heparina de baixo peso molecular para prevenção de eventos tromboembólicos e com antivirais, por dez dias. Cinco dias após o início dos sintomas neurológicos foi feito teste eletrodiagnóstico, que evidenciou desmielinização típica da síndrome de Guillain-Barré. Onze dias após a admissão, já com melhora do quadro, o paciente relatou parestesia em mãos, tetraparesia e arreflexias. Foi feita análise do liquor e outros exames à procura de outras causas para o quadro, mas não houve sucesso.

Assim sendo, pode-se observar que a síndrome de Guillain-Barré decorrente da infecção pelo SARS-CoV2 pode tanto preceder os sintomas típicos da COVID-19 como ocorrer dias após o início sintomático típico. $O$ tratamento é o mesmo feito em outras situações, baseando-se principalmente em imunoglobulinas e em antivirais (CAMDESSANCHE JP, et al., 2020; ZHAO H, et al., 2020).

\section{Paralisia facial periférica}

A paralisia facial periférica ocorre devido a lesão do nervo facial, sétimo par craniano que apresenta função mista. Esta patologia é caracterizada pela diminuição ou abolição, temporária ou não, da função do nervo facial em seu segmento periférico. Apresentando-se clinicamente por alterações da mobilidade dos músculos faciais, secreção salivar e lacrimal e sensibilidade facial, resultando em comprometimento estético e funcional aos indivíduos acometidos (DIB GC, et al., 2020).

Figueiredo R, et al. (2020) publicaram o caso de uma paciente de 35 anos, primigesta, com 39 semanas de gestação, que se apresentou ao departamento de emergência obstétrica com desvio progressivo da comissura labial esquerda, com dois dias de evolução. A paciente não apresentou outro sintoma associado à paralisia facial e seu teste foi positivo para COVID-19. A associação entre a paralisia e a infecção pelo SARSCoV-2 se dá em decorrência da já conhecida característica neurotrópica do vírus. A porcentagem de pacientes infectados que apresentaram alterações neurológicas periféricas foi de 8,9\%.

Homma Y, et al (2020) descreveram o caso de uma paciente de 35 anos com diagnóstico de COVID-19 e com diversos sintomas flu-like, como febre, tosse, dor de cabeça, etc. Além dos sintomas mais comuns da infecção pelo SARS-CoV-2, a paciente apresentou paralisia facial periférica direita, anosmia e ageusia do lado direito da língua.

Assim sendo, pode-se notar que a paralisia facial na COVID-19 pode ocorrer tanto em pacientes assintomáticos quanto naqueles que possuem sintomas típicos, e que a forma mais comum é a unilateral. $O$ tratamento realizado foi desde a administração de corticoides, unicamente, até o uso de antivirais (FIGUEIREDO R, et al., 2020; HOMMA Y, et al., 2020). 


\section{CONSIDERAÇÕES FINAIS}

Pressupõe-se que pacientes com acometimentos neurológicos pré-existentes sejam mais propensos a desenvolver complicações nervosas em função da infecção por Sars-Cov-2. Foram relatados cefaleia, vertigem, acidente vascular encefálico, paralisia facial periférica, alterações dos níveis de consciência, mielite transversa, encefalopatia hemorrágica aguda, encefalopatia, encefalite, ataxia, hipogeusia, hiposmia e neuralgia. Os mais frequentemente são: cefaleia, anosmia, ageusia e paresias. Há evidências que apontam para a relação da infecção por Sars-CoV-2 com a ocorrência da Síndrome de Guillain Barré e a Demência de Alzheimer. Mais estudos tornam-se necessários de modo a esclarecer a relação entre a COVID-19 e seus impactos no sistema nervoso, complicações imediatas e a longo prazo.

\section{REFERÊNCIAS}

1. ABDENNOUR L, et al. Interaction brain-lungs. In: Annales francaises d'anesthesie et de reanimation. 2012; 31(6): e101-e107.

2. BEYROUTI R, et al. Characteristics of ischaemic stroke associated with COVID-19. J Neurol Neurosurg Psychiatry. 2020;91(8):889891.

3. BOHMWALD K, et al. Neurologic alterations due to respiratory virus infections. Front Cell Neurosci. 2018; $12: 386$.

4. CAMDESSANCHE JP, et al. COVID-19 may induce Guillain-Barré syndrome. Rev Neurol (Paris). 2020; 176(6): 516-518.

5. $\mathrm{CHU} \mathrm{H}$, et al. Comparative tropism, replication kinetics, and cell damage profiling of SARS-CoV-2 and SARS-CoV with implications for clinical manifestations, transmissibility, and laboratory studies of COVID-19: an observational study. Lancet Microbe. 2020; 1(1): e14-e23.

6. DE FELICE FG, et al. Severe acute respiratory syndrome coronavirus 2 (SARS-CoV-2) and the central nervous system. Trends Neurosci. 2020; 43(6): 355-357.

7. DE SOUZA GOMES A, et al. Associação entre o COVID-19 e manifestações neurológicas. Brazilian Journal of Development,2020; 6 (11): 88950-88961.

8. DIB GC, et al. Paralisia facial periférica. Rev Bras Med, 2004; 61(3), 110-7.

9. ELLUL MA, et al. Neurological associations of COVID-19. Lancet Neurol. 2020;19(9):767-783.

10. ELLUL MA, SOLOMON T. Acute encephalitis - diagnosis and management. Clin Med. 2018; 18(2): 155-159.

11. FIGUEIREDO R, et al. Peripheral facial paralysis as presenting symptom of COVID-19 in a pregnant woman. BMJ Case Rep. 2020; 13(8): e237146.

12. GUAN WJ, et al. Clinical characteristics of coronavirus disease 2019 in China. N Engl J Med. 2020; 382(18): 1708-1720.

13. GUO YR, et al. The origin, transmission and clinical therapies on coronavirus disease 2019 (COVID- 19) outbreak - an update on the status. Mil Med Res. 2020; 7(1): 1-10.

14. HOMMA Y, et al. Coronavirus Disease-19 Pneumonia with Facial Nerve Palsy and Olfactory Disturbance. Intern Med. 2020; 59(14): $1773-1775$

15. JIN H, et al. Consensus for prevention and management of coronavirus disease 2019 (COVID-19) for neurologists. Stroke Vasc Neurol. 2020; 5(2): 146-151.

16. KOCHI AN, et al. Cardiac and arrhythmic complications in patients with COVID- 19. J Cardiovasc Electrophysiol. 2020; 31(5): 10031008.

17. LEE JM, LEE SJ. Olfactory and Gustatory Dysfunction in a COVID-19 Patient with Ankylosing Spondylitis Treated with Etanercept: Case Report. J Korean Med Sci. 2020; 35(21): e201.

18. LENNON JC. Neurologic and Immunologic Complications of COVID-19: Potential Long-Term Risk Factors for Alzheimer's Disease. J Alzheimers Dis Rep. 2020; 4(1): 217-221

19. LI Y, et al. Coronavirus neurovirulence correlates with the ability of the virus to induce proinflammatory cytokine signals from astrocytes and microglia. J Virol. 2004; 78(7): 3398-3406.

20. LING L, et al. Critically ill patients with COVID-19 in Hong Kong: a multicentre retrospective observational cohort study. Crit Care Resusc. 2020; 22(2): 119-125.

21. LOPES J, Complicações neurológicas em pacientes infectados por Sars-Cov-2 (COVID-19). Ações neurológicas em pacientes infectados por Sars-Cov-2 (COVID-19). 2020; 1(6): 388-416.

22. MAO L, et al. Neurological manifestations of hospitalized patients with COVID-19 in Wuhan, China: a retrospective case series study. MedRxiv. 2020.

23. MCGONAGLE D, et al. Immune mechanisms of pulmonary intravascular coagulopathy in COVID-19 pneumonia. Lancet Rheumatol. 2020; 2(7): e437-e445.

24. MEHTA P, et al. COVID-19: consider cytokine storm syndromes and immunosuppression. Lancet. 2020; 395(10229): $1033-1034$.

25. MONTALVAN V, et al. Neurological manifestations of COVID-19 and other coronavirus infections: A systematic review. Clin Neurol Neurosurg. 2020; 194: 105921.

26. MORIGUCHIT T, .et al. A first case of meningitis/encephalitis associated with SARS-Coronavirus-2. Int J Infect Dis. 2020; 94: 55-58.

27. MUNHOZ RP, et al. Neurological complications in patients with SARS-CoV-2 infection: a systematic review. Arq Neuropsiquiatr. 2020; 78(5): 290-300.

28. PAPA SM, et al. Impact of the COVID-19 pandemic on Parkinson's disease and movement disorders. Movement Disorders. 2020; 6(10): 1002.

29. PLEASURE SJ, et al. The spectrum of neurologic disease in the severe acute respiratory syndrome coronavirus 2 pandemic infection: neurologists move to the frontlines. JAMA Neurol. 2020; 77(6): 679-680.

30. SHARIFIAN-DORCHE M, et al. Neurological complications of coronavirus infection; a comparative review and lessons learned during the COVID-19 pandemic. J Neurol Sci. 2020; 417: 117085.

31. VAIRA LA, et al. In Response to Anosmia and Ageusia: Common Findings in COVID-19 Patients. Laryngoscope. 2020; 130 (11): e695.

32. VANDERVORST F, et al. Encephalitis associated with the SARS-CoV-2 virus: a case report. Interdiscip Neurosurg. 2020; $22: 100821$.

33. WANG Z, et al. COVID-19 Associated Ischemic Stroke and Hemorrhagic Stroke: Incidence, Potential Pathological Mechanism, and Management. Front Neurol. 2020; 11: 1152.

34. XIANG P, et al. First case of 2019 novel coronavirus disease with encephalitis. ChinaXiv. 2020; 03: 15.

35. ZHAO H, et al. Guillain-Barré syndrome associated with SARS-CoV-2 infection: causality or coincidence? Lancet Neurol. May 2020; 19(5): 383-384. 